\title{
Estimating stature in females by using the external ear morphometry
}

\author{
Srijith $^{1^{*}}$, Murugan $\mathbf{M}^{2}$ \\ ${ }^{\mathbf{1}}$ Assistant Professor, ${ }^{\mathbf{2}}$ Professor and HOD, ${ }^{\mathbf{1}, \mathbf{2}}$ Dept. of Forensic Medicine \& Toxicology, ${ }^{\mathbf{1 , 2}}$ Karpagam Faculty of Medical Sciences \& \\ Research, Coimbatore, Tamil Nadu, India
}

\begin{abstract}
Introduction: Identification refers to determining the individuality of a person. External ear has often emerged as a potential tool for forensic investigations and establishment of personal identification. The present study aims to estimate stature using external ear measurements in males thereby deriving at linear regression formulas and finding out the most reliable parameter.

Materials and Methods: The present study was conducted on 50 young and healthy female medical interns, within the age group $22-25$ years. Besides stature, eight external ear parameters, namely, ear length, ear width, lobule length, lobule width, conchal length, conchal width, tragus length and tragus width were considered and their measurements obtained from both the ears of each participant.

Results: Obtained data were analysed by SPSS software version 20.There was a strong significant positive correlation between all measurements and stature. Linear regression formulas for the eight parameters were derived. Actual stature and estimated stature from measurements of both ears were compared.

Conclusion: The study concluded that the morphometry of the external ear can be used in the estimation of the stature and that the conchal length was the most reliable parameter to estimate stature.
\end{abstract}

Keywords: Correlation coefficient, Ear, Identification, Regression equation, Stature.

\section{Introduction}

Stature refers to body height. It is one of the most important parameters to determine the physical identity of an individual. There is a definite biological relationship of stature with all the body parts such as head, trunk, extremities, vertebral column, etc. ${ }^{1-11}$

It is a known fact from many studies that stature bears a direct relation to the length of long bones, ${ }^{12-15}$ and linear regression formulas have been derived to estimate the stature from the length of long bones. These linear regression formulas are applied by the forensic experts to estimate the stature whenever skeletal remains are bought for examination.

Whenever fragmented or dismembered remains such as a decapitated head or only a portion of the face are bought for examination, it becomes a challenging task for a forensic expert to estimate the stature and thus establish the identity. In such scenarios, experts need to make an attempt to estimate stature from the cephalofacialdimensions ${ }^{16-25}$ such as length, breadth, and circumference of head, nasal morphometry and external ear morphometry. However, studies with respect to the above dimensions are scanty.

The present study aims to estimate stature by using external ear measurements thereby attempting to derive at a linear regression formula for each of the parameters and finding out the most reliable parameter to estimate stature among females.

\section{Materials and Methods}

The present study was conducted on 50 young and healthy female medical interns, within the age group 22-25 years. Subjects with external ear deformities (either congenital or acquired) were excluded from the study.

The stature or height is the distance between the highest point on the head (vertex) and floor. It was measured in the standing position by using a height measuring scale and the height was recorded in centimetres $(\mathrm{cm})$.

Standardized measurements of the external ear parameters were measured by manual verniercalipers and recorded in millimetres $(\mathrm{mm})$ according to the landmarked points defined by DeCarloet $\mathrm{al}^{26}$ and the methodology was adopted by Mckinney et $\mathrm{al}^{27}$ and Bucker et al. ${ }^{28}$

The parameters measured in the present study were:

1. Ear length (EL)

2. Ear width (EW)

3. Lobule length (LL)

4. Lobule width (LW)

5. Conchal length (CL)

6. Conchal width $(\mathrm{CW})$

7. Tragus length (TL)

8. Tragus width (TW)

All the measurements were taken thrice to ensure accuracy and the mean of the three readings was considered as the final reading. All measurements were carried out by the same investigator in order to minimize bias and error of identification of the parts of the external ear involved in the measurements.

\footnotetext{
*Corresponding Author: Srijith, Dept. of Forensic Medicine \& Toxicology, ${ }^{2}$ Karpagam Faculty of Medical Sciences \& Research, Coimbatore, Tamil Nadu, India

Email: srijithfun@gmail.com

http://doi.org/10.18231/J.IJFCM.2019.041
} 
The entire ear measurements were recorded in millimetres ( $\mathrm{mm})$, systematically tabulated and statistically analysed using SPSS version 20 to derive at a linear regression equation for estimation of stature. Correlation was calculated to assess the correlation between stature and external ear morphometry, and student $t$ test was applied to test the significance. $\mathrm{p}$ value $<0.05$ was considered significant.

\section{Results}

The stature of the female medical interns ranged from $146 \mathrm{~cm}-178 \mathrm{~cm}$. The mean values and standard deviation are depicted in Table1.
The descriptive statistics of the measurements of external ear (in $\mathrm{mm}$ ) along with stature using $\mathrm{t}$ test are tabulated in Table2.

The correlation between different parameters of both ears and stature is shown in Table3.

Multiple stepwise linear regression analysis of the left and right ear parameters to predict stature is shown in Table 4 and Table 5 respectively.

Table 6 shows the comparision between the actual stature and estimated stature from measeurements of both ears.

Table 1:

\begin{tabular}{|l|l|}
\hline & Females \\
\hline Stature range $(\mathrm{cm})$ & $146-178$ \\
\hline Mean+/-SD $(\mathrm{cm})$ & $162.47+/-8.7$ \\
\hline
\end{tabular}

Table 2:

\begin{tabular}{|c|c|c|c|}
\hline & LT side & RT side & p value \\
\hline EL(range) & $(51.80-75.70)$ & $(51.80-75.70)$ & \multirow{2}{*}{0.37} \\
\hline Mean \pm S.D & $63.73 \pm 7.21$ & $63.74 \pm 7.19$ & \\
\hline EW(range) & $(31.20-42.10)$ & $(31.30-42.10)$ & \multirow{2}{*}{0.29} \\
\hline Mean \pm S.D & $36.44 \pm 3.15$ & $36.45 \pm 3.13$ & \\
\hline LL(range) & $(13.60-27.90)$ & $(13.60-28.00)$ & \multirow{2}{*}{$0.017^{*}$} \\
\hline Mean \pm S.D & $20.28 \pm 4.08$ & $20.30 \pm 4.09$ & \\
\hline LW(range) & $(13.20-27.30)$ & $(13.10-27.40)$ & \multirow{2}{*}{$0.00 *$} \\
\hline Mean \pm S.D & $20.24 \pm 3.98$ & $20.29 \pm 4.00$ & \\
\hline CL(range) & $(20.60-37.90)$ & $(19.80-36.90)$ & \multirow{2}{*}{0.94} \\
\hline Mean \pm S.D & $27.34 \pm 4.33$ & $27.34 \pm 4.27$ & \\
\hline CW(range) & $(11.20-30.30)$ & $(11.0-30.0)$ & \multirow{2}{*}{0.85} \\
\hline Mean \pm S.D & $24.21 \pm 5.54$ & $24.21 \pm 5.52$ & \\
\hline TL(range) & $(10.20-17.20)$ & $(10.00-17.30)$ & \multirow{2}{*}{0.09} \\
\hline Mean \pm S.D & $12.88 \pm 1.58$ & $12.92 \pm 1.56$ & \\
\hline TW(range) & $(4.20-7.40)$ & $(4.20-7.50)$ & \multirow{2}{*}{0.22} \\
\hline Mean \pm S.D & $6.30 \pm 0.87$ & $6.27 \pm 0.85$ & \\
\hline
\end{tabular}

*significant

Table 2-EL:Ear

length,EW:Earwidth;LL:Lobulelength;LW:Lobulewidth;CL:Conchallength;CW:Conchalwidth;TL:Traguslength;TW:Tragus width; significant

Table 3:

\begin{tabular}{|c|c|c|c|}
\hline \multicolumn{2}{|c|}{} & r value & p value \\
\hline EL & Left & 0.97 & $0.00^{*}$ \\
\hline & Right & 0.97 & $0.00^{*}$ \\
\hline EW & Left & 0.93 & $0.00^{*}$ \\
\hline LL & Right & 0.93 & $0.00^{*}$ \\
\hline & Left & 0.95 & $0.00^{*}$ \\
\hline LW & Right & 0.95 & $0.00^{*}$ \\
\hline CL & Left & 0.94 & $0.00^{*}$ \\
\hline & Right & 0.94 & $0.00^{*}$ \\
\hline CW & Left & 0.93 & $0.00^{*}$ \\
\hline & Right & 0.94 & $0.00^{*}$ \\
\hline & Left & 0.91 & $0.00^{*}$ \\
\hline
\end{tabular}




\begin{tabular}{|c|c|c|c|}
\hline TL & Left & 0.92 & $0.00^{*}$ \\
\hline & Right & 0.92 & $0.00^{*}$ \\
\hline TW & Left & 0.95 & $0.00^{*}$ \\
\hline & Right & 0.95 & $0.00^{*}$ \\
\hline
\end{tabular}

*significant

r-correlation

Table 3-EL:Ear

length,EW:Earwidth;LL:Lobulelength;LW:Lobulewidth;CL:Conchallength;CW:Conchalwidth;TL:Traguslength;TW:Tragus width;

Table 4:

\begin{tabular}{|c|c|c|c|c|c|c|}
\hline & $\mathrm{B}$ & SEM & $r$ & SEE & $\mathrm{p}$ value & Regression Equation \\
\hline $\begin{array}{l}\text { Left EL } \\
\text { constant }\end{array}$ & $\begin{array}{c}1.17 \\
87.51\end{array}$ & $\begin{array}{c}0.051 \\
3.17 \\
\end{array}$ & 0.98 & 1.98 & $0.00^{*}$ & $\mathrm{~S}=87.51+(1.17 \times$ Left EL $)$ \\
\hline $\begin{array}{l}\text { Left EL } \\
\text { Left EW } \\
\text { constant }\end{array}$ & $\begin{array}{r}1.55 \\
-0.89 \\
95.71\end{array}$ & $\begin{array}{l}0.23 \\
0.53 \\
5.85\end{array}$ & 0.97 & 1.92 & $\begin{array}{l}0.00^{*} \\
0.105\end{array}$ & $\begin{array}{c}S=95.71+(1.55 \times \text { Left EL }) \\
S=95.71+(-0.89 \times \text { Left EW })\end{array}$ \\
\hline $\begin{array}{l}\text { Left EL } \\
\text { Left EW } \\
\text { Left LL } \\
\text { constant }\end{array}$ & $\begin{array}{c}1.23 \\
-0.94 \\
0.64 \\
105.34\end{array}$ & $\begin{array}{l}0.25 \\
0.49 \\
0.27 \\
6.77 \\
\end{array}$ & 0.98 & 1.78 & $\begin{array}{c}0.00^{*} \\
0.06 \\
0.026 *\end{array}$ & $\begin{array}{c}S=105.34+(1.23 \times \text { Left EL }) \\
S=105.34+(-0.4 \times \text { Left } E W) \\
S=105.34+(0.64 \times \text { Left } L L)\end{array}$ \\
\hline $\begin{array}{l}\text { Left EL } \\
\text { Left EW } \\
\text { Left LL } \\
\text { Left LW } \\
\text { constant }\end{array}$ & $\begin{array}{c}1.23 \\
-0.90 \\
0.34 \\
0.27 \\
104.25\end{array}$ & $\begin{array}{l}0.26 \\
0.51 \\
0.93 \\
0.81 \\
7.62\end{array}$ & 0.98 & 1.81 & $\begin{array}{c}0.00^{*} \\
0.09 \\
0.71 \\
0.74\end{array}$ & $\begin{array}{c}S=104.25+(1.23 \times \text { Left EL }) \\
S=104.25+(-0.90 \times \text { Left EW }) \\
S=104.25+(0.34 \times \text { Left } L L) \\
S=104.25+(0.27 \times \text { Left } L W)\end{array}$ \\
\hline $\begin{array}{l}\text { Left EL } \\
\text { Left EW } \\
\text { Left LL } \\
\text { Left LW } \\
\text { Left CL } \\
\text { constant }\end{array}$ & $\begin{array}{c}1.24 \\
-0.91 \\
0.36 \\
0.26 \\
-0.01 \\
104.23\end{array}$ & $\begin{array}{l}0.30 \\
0.53 \\
1.00 \\
0.86 \\
0.28 \\
7.78\end{array}$ & 0.98 & 1.84 & $\begin{array}{c}0.00^{*} \\
0.10 \\
0.71 \\
0.76 \\
0.95\end{array}$ & $\begin{array}{c}S=104.23+(1.26 \times \text { Left EL }) \\
S=104.23+(-0.91 \times \text { Left } E W) \\
S=104.23+(0.36 \times \text { Left } L L) \\
S=104.23+(0.26 \times \text { Left } L W) \\
S=104.23+(-0.01 \times \text { Left } C L)\end{array}$ \\
\hline $\begin{array}{l}\text { Left EL } \\
\text { Left EW } \\
\text { Left LL } \\
\text { Left LW } \\
\text { Left CL } \\
\text { Left CW } \\
\text { constant }\end{array}$ & $\begin{array}{c}1.16 \\
-1.08 \\
0.42 \\
0.06 \\
0.13 \\
0.27 \\
111.26\end{array}$ & $\begin{array}{c}0.29 \\
0.51 \\
0.96 \\
0.83 \\
0.28 \\
0.15 \\
8.4 \\
\end{array}$ & 0.98 & 1.76 & $\begin{array}{c}0.001 * \\
0.047 * \\
0.66 \\
0.93 \\
0.64 \\
0.08\end{array}$ & $\begin{array}{c}S=112.52+(1.13 \times \text { Left EL }) \\
S=112.52+(-1.17 \times \text { Left } E W) \\
S=112.52+(0.64 \times \text { Left } L L) \\
S=112.52+(-0.11 \times \text { Left } L W) \\
S=112.52+(0.11 \times \text { Left } C L) \\
S=112.52+(0.27 \times \text { Left } C W)\end{array}$ \\
\hline $\begin{array}{l}\text { Left EL } \\
\text { Left EW } \\
\text { Left LL } \\
\text { Left LW } \\
\text { Left CL } \\
\text { Left CW } \\
\text { Left TL } \\
\text { constant }\end{array}$ & $\begin{array}{c}1.00 \\
-0.95 \\
0.82 \\
-0.31 \\
-0.25 \\
0.24 \\
1.38 \\
106.16\end{array}$ & $\begin{array}{l}0.26 \\
0.46 \\
0.86 \\
0.75 \\
0.29 \\
0.13 \\
0.50 \\
7.67\end{array}$ & 0.98 & 1.56 & $\begin{array}{c}0.001 * \\
0.051 \\
0.35 \\
0.68 \\
0.38 \\
0.09 \\
0.012 *\end{array}$ & $\begin{array}{c}S=106.16+(1.00 \times \text { Left EL }) \\
S=106.16+(-0.95 \times \text { Left } E W) \\
S=106.16+(0.82 \times \text { Left } L L) \\
S=106.16+(-0.31 \times \text { Left } L W) \\
S=106.16+(-0.25 \times \text { Left } C L) \\
S=106.16+(0.24 \times \text { Left } C W) \\
S=106.16+(1.38 \times \text { Left } T L)\end{array}$ \\
\hline $\begin{array}{c}\text { Left EL } \\
\text { Left EW } \\
\text { Left LL } \\
\text { Left LW } \\
\text { Left CL } \\
\text { Left CW } \\
\text { Left TL } \\
\text { Left TW } \\
\text { constant }\end{array}$ & $\begin{array}{c}0.69 \\
-0.59 \\
0.30 \\
0.10 \\
-0.11 \\
-0.12 \\
1.19 \\
3.83 \\
98.10\end{array}$ & $\begin{array}{l}0.21 \\
0.36 \\
0.67 \\
0.58 \\
0.22 \\
0.13 \\
0.39 \\
0.94 \\
6.21\end{array}$ & 0.99 & 1.19 & $\begin{array}{c}0.004^{*} \\
0.12 \\
0.65 \\
0.86 \\
0.60 \\
0.38 \\
0.006^{*} \\
0.001^{*}\end{array}$ & $\begin{array}{c}S=97.74+(0.69 \times \text { Left EL }) \\
S=97.74+(-0.54 \times \text { Left } E W) \\
S=97.74+(0.48 \times \text { Left } L L) \\
S=97.74+(-0.09 \times \text { Left LW }) \\
S=97.74+(-0.03 \times \text { Left } C L) \\
S=97.74+(-0.11 \times \text { Left } C W) \\
S=97.74+(0.89 \times \text { Left TL }) \\
S=97.74+(3.88 \times \text { Left TW })\end{array}$ \\
\hline
\end{tabular}

*significant 
B-constant derived;r-correlation;SEM-standard error of measurement;SEE-standard error of estimate

Table 4-EL:Ear length,

EW:Earwidth;LL:Lobulelength;LW:Lobulewidth;CL:Conchallength;CW:Conchalwidth;TL:Traguslength;TW:Tragus width;

Table 5:

\begin{tabular}{|c|c|c|c|c|c|c|c|}
\hline & B & SEM & $\mathrm{r}$ & & SEE & $\mathrm{p}$ value & Regression Equation \\
\hline $\begin{array}{c}\text { Right EL } \\
\text { constant }\end{array}$ & $\begin{array}{c}1.17 \\
87.35\end{array}$ & $\begin{array}{c}0.051 \\
3.29\end{array}$ & 0.97 & 0.95 & 1.99 & $0.00 *$ & $S=87.35+(1.17 \times$ Right EL $)$ \\
\hline $\begin{array}{l}\text { Right EL } \\
\text { Right EW } \\
\text { constant }\end{array}$ & $\begin{array}{r}1.58 \\
-0.95 \\
96.20 \\
\end{array}$ & $\begin{array}{l}0.23 \\
0.53 \\
5.92\end{array}$ & 0.97 & 0.95 & 1.91 & $\begin{array}{c}0.00^{*} \\
0.08\end{array}$ & $\begin{array}{c}S=96.20+(1.58 \times \text { Right EL }) \\
S=96.20+(-0.95 \times \text { Right EW })\end{array}$ \\
\hline $\begin{array}{l}\text { Right EL } \\
\text { Right EW } \\
\text { Right LL } \\
\text { constant }\end{array}$ & $\begin{array}{c}1.25 \\
-1.01 \\
0.65 \\
106.15\end{array}$ & $\begin{array}{l}0.25 \\
0.49 \\
0.27 \\
6.84\end{array}$ & 0.98 & 0.96 & 1.76 & $\begin{array}{c}0.00^{*} \\
0.052 \\
0.023 *\end{array}$ & $\begin{array}{c}S=106.15+(1.25 \times \text { Right EL }) \\
S=106.15+(-1.01 \times \text { Right } E W) \\
S=106.15+(0.65 \times \text { Right } L L)\end{array}$ \\
\hline $\begin{array}{l}\text { Right EL } \\
\text { Right EW } \\
\text { Right LL } \\
\text { Right LW } \\
\text { constant }\end{array}$ & $\begin{array}{c}1.25 \\
-1.00 \\
0.58 \\
0.07 \\
105.8\end{array}$ & $\begin{array}{l}0.26 \\
0.52 \\
0.90 \\
0.78 \\
7.72\end{array}$ & 0.98 & 0.96 & 1.80 & $\begin{array}{c}0.00^{*} \\
0.06 \\
0.52 \\
0.92\end{array}$ & $\begin{array}{c}S=105.8+(1.25 \times \text { Right } E L) \\
S=105.8+(-1.00 \times \text { Right } E W) \\
S=105.8+(0.58 \times \text { Right } L L) \\
S=105.8+(0.07 \times \text { Right } L W)\end{array}$ \\
\hline $\begin{array}{l}\text { Right EL } \\
\text { Right EW } \\
\text { Right LL } \\
\text { Right LW } \\
\text { Right CL } \\
\text { constant }\end{array}$ & $\begin{array}{c}1.26 \\
-1.00 \\
0.60 \\
0.05 \\
-0.02 \\
105.8 \\
\end{array}$ & $\begin{array}{l}0.31 \\
0.54 \\
0.96 \\
0.82 \\
0.30 \\
7.88 \\
\end{array}$ & 0.98 & 0.96 & 1.83 & $\begin{array}{c}0.00^{*} \\
0.076 \\
0.54 \\
0.94 \\
0.94\end{array}$ & $\begin{array}{c}S=105.8+(1.26 \times \text { Right EL }) \\
S=105.8+(-1.00 \times \text { Right } E W) \\
S=105.8+(0.60 \times \text { Right } L L) \\
S=105.8+(0.05 \times \text { Right } L W) \\
S=105.8+(-0.02 \times \text { Right } C L)\end{array}$ \\
\hline $\begin{array}{l}\text { Right EL } \\
\text { Right EW } \\
\text { Right LL } \\
\text { Right LW } \\
\text { Right CL } \\
\text { Right CW } \\
\text { constant }\end{array}$ & $\begin{array}{c}1.13 \\
-1.17 \\
0.64 \\
-0.11 \\
0.11 \\
0.27 \\
112.52 \\
\end{array}$ & $\begin{array}{l}0.30 \\
0.52 \\
0.92 \\
0.79 \\
0.30 \\
0.14 \\
8.30\end{array}$ & 0.98 & 0.96 & 1.75 & $\begin{array}{c}0.001 * \\
0.035^{*} \\
0.49 \\
0.88 \\
0.70 \\
0.07\end{array}$ & $\begin{array}{c}S=112.52+(1.13 \times \text { Right EL }) \\
S=112.52+(-1.17 \times \text { Right } E W) \\
S=112.52+(0.64 \times \text { Right } L L) \\
S=112.52+(-0.11 \times \text { Right } L W) \\
S=112.52+(0.11 \times \text { Right } C L) \\
S=112.52+(0.27 \times \text { Right } C W)\end{array}$ \\
\hline $\begin{array}{l}\text { Right EL } \\
\text { Right EW } \\
\text { Right LL } \\
\text { Right LW } \\
\text { Right CL } \\
\text { Right CW } \\
\text { Right TL } \\
\text { constant }\end{array}$ & $\begin{array}{c}1.01 \\
-1.06 \\
0.98 \\
-0.47 \\
-0.15 \\
0.26 \\
1.19 \\
108.46 \\
\end{array}$ & $\begin{array}{l}0.27 \\
0.47 \\
0.84 \\
0.72 \\
0.29 \\
0.13 \\
0.48 \\
7.68\end{array}$ & 0.98 & 0.97 & 1.58 & $\begin{array}{c}0.001 * \\
0.036^{*} \\
0.25 \\
0.52 \\
0.60 \\
0.06 \\
0.021 *\end{array}$ & $\begin{array}{c}S=108.46+(1.01 \times \text { Right } E L) \\
S=108.46+(-1.06 \times \text { Right } E W) \\
S=108.46+(0.98 \times \text { Right } L L) \\
S=108.46+(-0.47 \times \text { Right } L W) \\
S=108.46+(0.15 \times \text { Right } C L) \\
S=108.46+(0.276 \times \text { Right } C W) \\
S=108.46+(1.19 \times \text { Right } T L)\end{array}$ \\
\hline $\begin{array}{l}\text { Right EL } \\
\text { Right EW } \\
\text { Right LL } \\
\text { Right LW } \\
\text { Right CL } \\
\text { Right CW } \\
\text { Right TL } \\
\text { Right TW } \\
\text { constant }\end{array}$ & $\begin{array}{c}0.69 \\
-0.54 \\
0.48 \\
-0.09 \\
-0.03 \\
-0.11 \\
0.89 \\
3.88 \\
97.74\end{array}$ & $\begin{array}{l}0.23 \\
0.40 \\
0.68 \\
0.59 \\
0.23 \\
0.14 \\
0.39 \\
1.06 \\
6.80\end{array}$ & 0.99 & 0.98 & 1.26 & $\begin{array}{c}0.008^{*} \\
0.19 \\
0.49 \\
0.88 \\
0.88 \\
0.44 \\
0.03^{*} \\
0.001^{*}\end{array}$ & $\begin{array}{c}S=97.74+(0.69 \times \text { Right } E L) \\
S=97.74+(-0.54 \times \text { Right } E W) \\
S=97.74+(0.48 \times \text { Right } L L) \\
S=97.74+(-0.09 \times \text { Right } L W) \\
S=97.74+(-0.03 \times \text { Right } C L) \\
S=97.74+(-0.11 \times \text { Right } C W) \\
S=97.74+(0.89 \times \text { Right } T L) \\
S=97.74+(3.88 \times \text { Right TW })\end{array}$ \\
\hline
\end{tabular}

*significant

B-constant derived;r-correlation;SEM-standard error of measurement;SEE-standard error of estimate

Table 5-EL:Ear

length,EW:Earwidth;LL:Lobulelength;LW:Lobulewidth;CL:Conchallength;CW:Conchalwidth;TL:Traguslength;TW:Tragus width; 
Table 6:

\begin{tabular}{|c|c|c|c|}
\hline & & Left & Right \\
\hline \multirow{2}{*}{ EL } & Range & $(146.92-174.33)$ & $(147.91-175.91)$ \\
\hline & Mean \pm S.D & $160.61 \pm 8.27$ & $161.93 \pm 8.42$ \\
\hline \multirow{2}{*}{ EW } & Range & $\begin{array}{l}(148.73-177.11) \\
\end{array}$ & $(148.78-176.75)$ \\
\hline & Mean \pm S.D & $162.25 \pm 8.14$ & $162.13 \pm 8.13$ \\
\hline \multirow{2}{*}{ LL } & Range & $(148.71-177.74)$ & $(147.82-17686)$ \\
\hline & Mean \pm S.D & $162.28 \pm 8.28$ & $162.32 \pm 8.20$ \\
\hline \multirow{2}{*}{ LW } & Range & $(148.73-177.97)$ & $(147.57-176.89)$ \\
\hline & Mean \pm S.D & $162.32 \pm 8.30$ & $162.32 \pm 8.20$ \\
\hline \multirow{2}{*}{ CL } & Range & $(149.78-182.31)$ & $(147.95-180.78)$ \\
\hline & Mean \pm S.D & $162.47 \pm 8.15$ & $162.43 \pm 8.21$ \\
\hline \multirow{2}{*}{$\mathrm{CW}$} & Range & $(143.80-171.11)$ & $(143.70-171.25)$ \\
\hline & Mean \pm S.D & $162.41 \pm 7.92$ & $162.86 \pm 8.00$ \\
\hline \multirow{2}{*}{$\mathrm{TL}$} & Range & $(148.75-184.31)$ & $(147.41-184.78)$ \\
\hline & Mean \pm S.D & $162.27 \pm 8.06$ & $162.39 \pm 8.02$ \\
\hline \multirow{2}{*}{ TW } & Range & (197.41- 266.46) & $(142.07-174.38)$ \\
\hline & Mean \pm S.D & $225.09 \pm 14.83$ & $162.40 \pm 8.32$ \\
\hline \multirow{2}{*}{ Actual stature } & Range & \multirow{2}{*}{\multicolumn{2}{|c|}{ (146-178) }} \\
\hline & Mean \pm S.D & & $162.47 \pm 8.7$ \\
\hline
\end{tabular}

Table 6-EL:Ear

length,EW:Earwidth;LL:Lobulelength;LW:Lobulewidth;CL:Conchallength;CW:Conchalwidth;TL:Traguslength;TW:Tragus width;

\section{Discussion}

The earliest reference to individuality of the external ear was made by Bertillon ${ }^{29}$ who mentioned in his book that it is almost impossible to meet with two ears which are identical in all their parts. Though Bertillon did not undertake any research work on the use of ear as personal identification tool, he had made the above observation from his experience gathered from working on anthroposcopy and anthropometry of criminals.

The identity of Veerappan, the notorious sandalwood smuggler in India who was killed by the Special Task Force in 2004 was at first established through the morphology of ear. While comparing the anatomical structure of Veerappan's external ear in antemortem and post-mortem photographs, the Forensic Scientist confirmed his identity on the basis of combination of various features. A large and squarish lobule with a flat tragus which is contiguous with the curved portion of the helix made Veerappan's ear unique, thus helping in his identification. ${ }^{30}$

The ear is more coplanar, less affected by ageing, unaffected by change of facial expression, though hair and ear ornaments can occlude its appearance. It is stated that the full length of the ear is reached by age 15 in males and age 13 in females. ${ }^{31}$ The external ear does suffer from a few limitations. After the age of 60 , the lobule continues to elongate contributing to the overall length of the ear. ${ }^{32}$

In a similar study conducted by Magaji Garba Tauraet $\mathrm{al}^{17}$, ear index, a new parameter was considered as one of the variables to predict stature. Ear index was calculated by era width/ear lengthx100. However, the study observed the least correlation in ear index and the highest correlation in ear width.

\section{Conclusion}

Estimation of stature is considered an important parameter in medicolegal and forensic examinations.

The present study concludes that the external ear morphometry is an additional important tool useful in the estimation of stature by using statistical methods, and conchal length being the most reliable parameter. The regression equations generated from the external ear measurements can be a supplementary approach for the estimation of stature when the extremities are not available. Also, the regression formula derived in this study will be of potential use in clinical, medicolegal and anthropological studies. The authors plan to conduct a similar study on male subjects too. The authors recommend that more such studies need to be conducted on both sexes in different regions involving all age groups.

\section{Acknowledgements}

The authors are grateful to Dr. Deepa B, Assistant Professor, Dept. of Public Health Dentistry, BIDS, Bangalore for helping us with the statistical analysis in the study.

\section{Ethical Clearance}

Issued by the Institutional Ethics Committee

\section{Consent}

Informed consent taken from medical interns for the study

\section{Conflict of interest}

None. 


\section{Source of Funding}

Self

\section{References}

1. Nagesh KR, Kumar GP. Estimation of stature from vertebral column length inSouth Indians. Leg Med (Tokyo) 2006;8:26972

2. Rastogi P, Nagesh KR, Yoganarasimha K. Estimation of stature from handdimensions of North and South Indians. Leg Med (Tokyo) 2008;10:185-9.

3. Nachiket S, Sujatha N, Priya R, Raveendranath V, Rema D, Roopa R et al. Reliability ofinter-anterior superior iliac spinous distance as compared to foot length for stature estimation in South Indians. J Forensic Leg Med 2010;17:352-4.

4. Mohanty SP, Babu SS, Nair NS. The use of arm span as a predictor of height: a study of South Indian women. J Orthop Surg (Hong Kong) 2001;9:19-23.

5. Rao PPJ, Sowmya J, Yoganarasimha K, Menezes RG, Kanchan T, Aswinidutt $R$ et al. Estimation of stature from cranial sutures in a South Indian male population. Int J Legal Med 2009;123:271-6

6. Krishan K, Sharma A. Estimation of stature from dimensions of hands and feet in a North Indian population. J Forensic Leg Med 2007;14:327-32.

7. Agnihotri AK, Purwar B, Googoolye K, Agnihotri S, Jeebun N. Estimation of stature by foot length. J Forensic Leg Med 2007;14:279-83.

8. Asha KR and Laxmi Prabha R. Estimation of stature from cephalic dimensions in Indian population. Anatomica Karnataka 2011;5(1)1-5.

9. IIayperuma I. On prediction of personal stature from cranial dimensions. Int J Morphol 2010;28(4):1135-40.

10. SeemaMahajan A. Estimation of Personal Height from the Length of Head in Punjab Zone. Int J Plant, Animal Environ Sci 2011;1(3):205-8.

11. Jadhav HR and Shah GV. Determination of personal height from length of head in Gujarat region. $J$ Anatomical Soc India 2004;53(1);20-1.

12. Smith SL. Stature Estimation of 3-10 year old children from long bone lengths. J Forensic Sci 2007;52;538-46.

13. Srijith, Jaffar Hussain AP, Kumar V, Santhosh CS, SK Mohanty. Estimation of stature from the length of the sternum:an autopsy based study. J Indian Acad Forensic Med 2018;40(1):80-4

14. Chandran M, Kumar V. Reconstruction of femur length from its fragments in South Indian Males. J Forensic Leg Med 2012;19:132-36

15. Kamal R, Yadav PK. Estimation of stature from different anthropometric measurements in kori population of NorthIndia. Egypt J Forensic Sci 2016;6(4):468-77

16. Abdelaleem S A. Fouad Abdelbaky F A. Estimation of stature in upper Egypt population from external ear morphometry. Int J Forensic Sci Pathol 4(10):276-85
17. Taura MG, Lawan HA, Abdullahi G, Musa HM. Height prediction from external ear morphometry:a pilot study. Int $J$ Res Health Sci 2016;4(1):15-9.

18. Swami S, Kumar M. Patnaik VVG. Estimation of stature from facial anthropometric measurements in 800 adult Haryanvi baniyas. Int J Basic Appl Med Sci 2015;5(1):122-32

19. Agnihotri AK, Smita K, Krishna G, Anishta A. Estimation of stature from cephalofacial dimensions by regression analysis in Indo Mauritian population. J Forensic Legal Med 2011;18:167-72.

20. Krishan K. Estimation of stature from cephalofacial anthropometry in North India population. Forensic Sci Int 2008;181(1-3):52-6.

21. Krishan K, Kumar R. Determination of stature from cephalo facial dimensions in a North India population. Legal Med (Tokyo) 2007;9(3):128-33.

22. Pelin C, Zagyapan R, Yazici C. Body height estimation from head and face dimensions:a different method. J Forensic Sci 2010;55(5):1326-30.

23. Ahmed AA. Cephalo facial analysis to estimate stature in Sudanessepopulation. Legal Med 2016;20:80-6.

24. Colmenares GG., Sanabria C. Medina, Liliana Carolina Baez. Estimation of stature by cephalometric facial dimensions in skeletonized bodies: study from a sample modern Colombians skeletal re, mains. Forensic Sci Int 2016;258(6):101-06.

25. Deopa D, Thakkar HK, Chandra Prakash, Niranjan R. Anthropometric measurements of external ear of medical students in Uttarkhand Region. J Anatomical Soc India 2013;62(1):79-83.

26. De Carlo D, Metaxas D, Stone M. An anthropometric face model using variational techniques. Proceedings of the 25th Annual Conference on Computer Graphics and Interactive Techniques New York. ACM. 1998;67-74.

27. McKinney P, Giese S, Placik O. Management of the ear in rhytidectomy. Plast Reconstr Surg 1993;92(5): 858-66.

28. Brucker MJ, Patel J, Sullivan PK. A morphometric study of the external ear: age and sex related differences. Plast Reconstr Surg 2003;112(2):647-52.

29. Bertillon A. Signaletic Instructions including the Theory and Practice of Anthropometrical Identification [R.W. McClaughry translation]. London: The Werner Company.1896.

30. Purakit R. Role of external ear in establishing personal identity-A short review. Austin J Forensic Sci Criminology 2015;2(2):1-5.

31. Skaria K, Alexander, David JS, Sivakumar B, Kang N. Amporphometric study of the human ear. J Plastic Reconstr Aesthetic Surg 2011;(64).41-47.

32. Purakit R. External Ear: Ananlaysis of its uniqueness. Egyptian J Forensic Sci 2016;(6):99-107.

How to cite this article: Srijith, Murugan M. Estimating stature in females by using the external ear morphometry. Indian J Forensic Community Med 2019;6(3):182-7. 